\title{
Achieving Agile C2 by Applying the Model Driven Architecture to C2 System Development
}

\author{
DUANMU Zhu-yun ${ }^{1, \text { a }}$ \\ ${ }^{1}$ Science and technology on information systems engineering laboratory, Nanjing 210007, China \\ a fairydm@qq.com
}

Keywords: agility; command and control system; Model Driven Architecture

\begin{abstract}
.
In order to meet the requirement of agility in Command and Control system, a concept model and a development pattern of agile $\mathrm{C} 2$ system are proposed. The core characters of agile $\mathrm{C} 2$ system are operations mission driven and the cooperative structure adjusting of the system resources. A building method of agile $\mathrm{C} 2$ system is proposed by using the model driven architecture, the overall framework is also proposed. Finally, the key techniques and the realization methods of agile C2 system are described.
\end{abstract}

\section{Introduction}

"Agile" has become one of the most important characteristics of the information age organization. it is a prerequisite for the successful response to the diverse and complex battlefield environment. In recent years domestic and foreign researchers have introduced Agility Research into military field. Among them, the most representative researcher is Dr. David S. Alberts. In the literature [1] "Agility advantage", he provides a conceptual framework, a set of metrics, and the results of a series of experiments to enable readers to understand agility of their organizations. From this time, the experts of military field began to do the research of $\mathrm{C} 2$ agility. From the existing public literatures, foreign experts studied in the $\mathrm{C} 2$ organization effectiveness measurement, agile $\mathrm{C} 2$ organization theory, $\mathrm{C} 2$ organization agility measurement methods. The magnum opus is "Agility Quotient" of Dr. David S. Albert, around "how to measure an organization's agility?" and "what is the need to have the agility?" [2] is discussed. Domestic scholars' research on agility is mainly focused on the research of agile C2 organization, and the research on the structure design and adjustment method of $\mathrm{C} 2$ in University of National Defense Technology. The paper studies the modeling, structure design and structure adjustment of agile $\mathrm{C} 2$ in the literature [3].

In general, the domestic and foreign scholars on the "agile" research focus on the research of the C2 organization, the study of the agile C2 system is still weak. The C2 system is the most basic material base of information warfare, is the "multiplier" of operation effectiveness. [5] Therefore, C2 system also needs to have the "agile" characteristics, to achieve flexible recomposition and reconfiguration, supporting system dynamic tailoring, integration and restructuring needs, in order to deal with the complex battlefield environment. Aimed at requirement of Command and Control system agility, the agile $\mathrm{C} 2$ system concept model and application pattern, and method of agile $\mathrm{C} 2$ system construction are studied in this paper.

\section{Basic Concepts}

According to the definition of the U.S. Department of Defense (CCRP) (command and control research project), agility is the capability to successfully effect, cope with and/or exploit changes in circumstances. Change is an essential prerequisite for agility. Without change entities do not need to be agile to be successful. Agility explicitly requires changes - the ability to deal with a dynamic situation. In situations that are stable or change in ways that do not have a significant impact on $\mathrm{C} 2$ system, C2 system does not need agility to be successful. Therefore, the construction of agile C2 system is based on the idea of "design for change". The basic method is to separate and wrap the 
changing system resources, and create a mapping from the diverse operations mission to the system resource workflow.

The construction and operation of the agile $\mathrm{C} 2$ system focus on the dynamic cooperation between the system resources, in order to improve the flexibility of the $\mathrm{C} 2$ system. The core characters of which are operations mission driven and the system resource cooperative structure adjusting. Operations mission driven is the input of the various operations mission, through the reasonable choice of the grid network distribution system resources, and optimize the structure and relationship between resources to meet the operational requirements of the stability of the system. Cooperative structure adjustment is to maintain good overall effectiveness in the face of the fierce changes in the internal state of the operations mission and system resources.

\section{Conceptual model of agile $\mathrm{C} 2$ system}

C2 system agility means in a fast and dynamic network environment, C2 system can organize different system resource in grid fast and dynamic, which can form system capability that meets operations mission needs in right time, right node and right function. which can be characterized by a variety of operations missions, organize different system resource fast and dynamic to response to the non-expected, complex and changeable battlefield environment successful.

The construction and operation of agile command system includes three stages: first, the mapping relationship between system resources and the system resource is established according to the system resources and the need to complete the mission. The second stage is to establish the system resource workflow according by cooperate relationship; the third stage is to maintain the overall effectiveness of the system.

In the development phase, the system resources are wrapped in accordance with the standards and protocols, and the change of system resources are wrapped and isolation, the impact of the entire system to the minimum. In addition, resources can be found and coordinated in the operating period, even after the deployment of the system, we can still use the new and better resources to replace the old and poor resources, improve system performance. And, according to the operations mission, the resource workflow can find the system resources to meet the needs and integrate them into a complete system in running time. it can be automatically reassembled or configuration system in a very short time, and the resources can be changed to meet the expected mission and complex and changeable battlefield environment.

\section{Application mode of agile charge system}

The application mode of agile charge system is shown in figure 1. The different organizations of the grid network wide area distribution will be packaged according to certain standards and protocols, submit to the whole army test, integration environment, the whole army test, integration environment will develop a series of strict standards for the submission of system resources for testing and integration, and feedback to the resource developers. Resource developers in accordance with feedback to modify the number of iterations after the test, the integration environment released to the military resources registration center for operation forces found and used. According to the operations mission of the higher level, the dynamic mapping method of the resource workflow is based on the dynamic mapping method of the resource workflow. After the completion of operation missions, operation forces will be the use of system resources feedback to the center of the military resources registration, and then from the army resource registration center feedback to the test, integrated environment for testing analysis, and will modify the views of the final feedback to the resource developers to modify, update the version of the submitted and tested. 


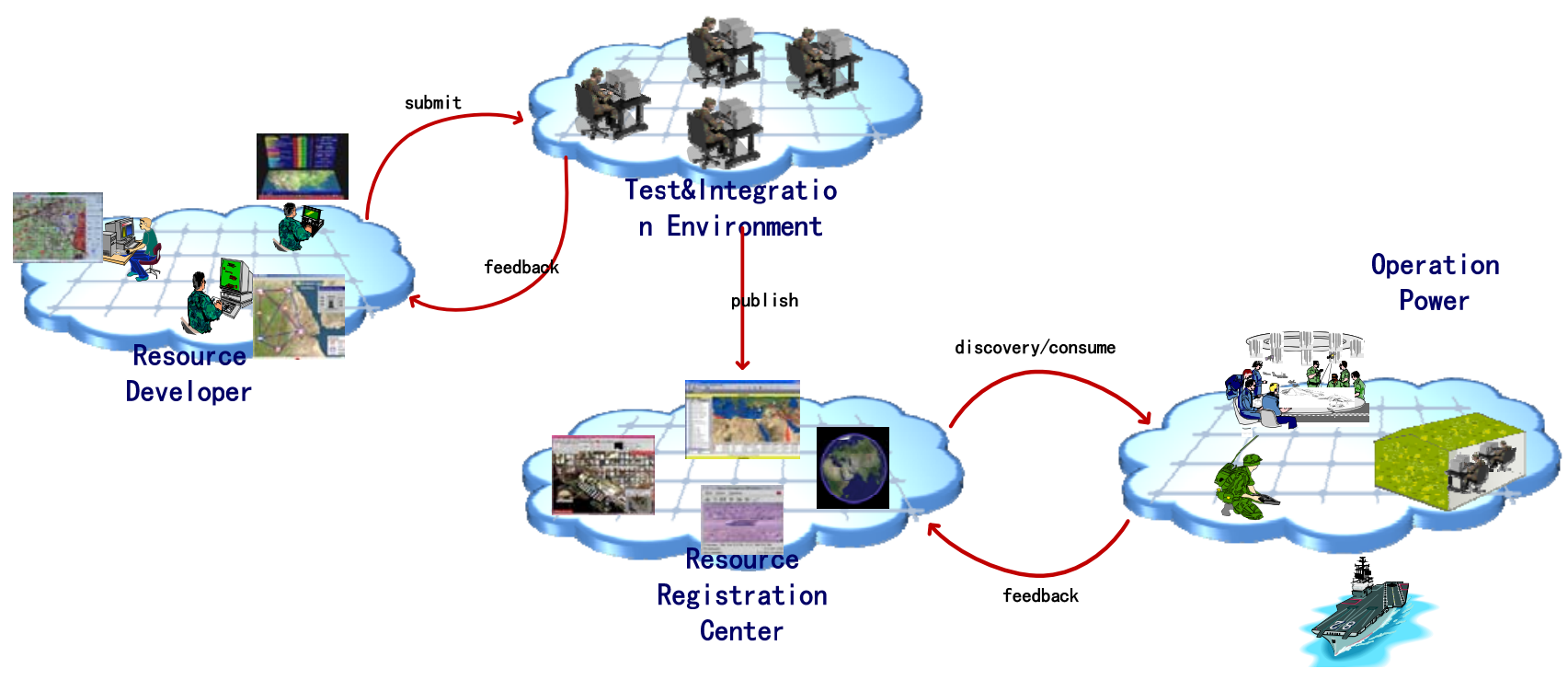

Fig. 1 The application mode of agile charge system

\section{A model driven approach to the construction of agile command system}

The overall framework of the model driven agile C2 system is shown in Figure 2, which includes six parts: requirements analysis, modeling, design, implementation, testing and verification, operation and maintenance. Requirements analysis stage collect and analyze business requirements; Modeling and designing stage model based on business requirements, design resource cooperate process based on system architecture model and resource cooperate architecture model, and design for resource optimize rule, system dynamic evolvement rule; In the implementation stage, the operations mission to the system resource cooperative workflow mapping is built based on operation mission to system meta-mission mapping rules; Testing and verification stage test and validate resource cooperate workflow; operation and maintenance stage integrate optimum resource to meet the operation mission.

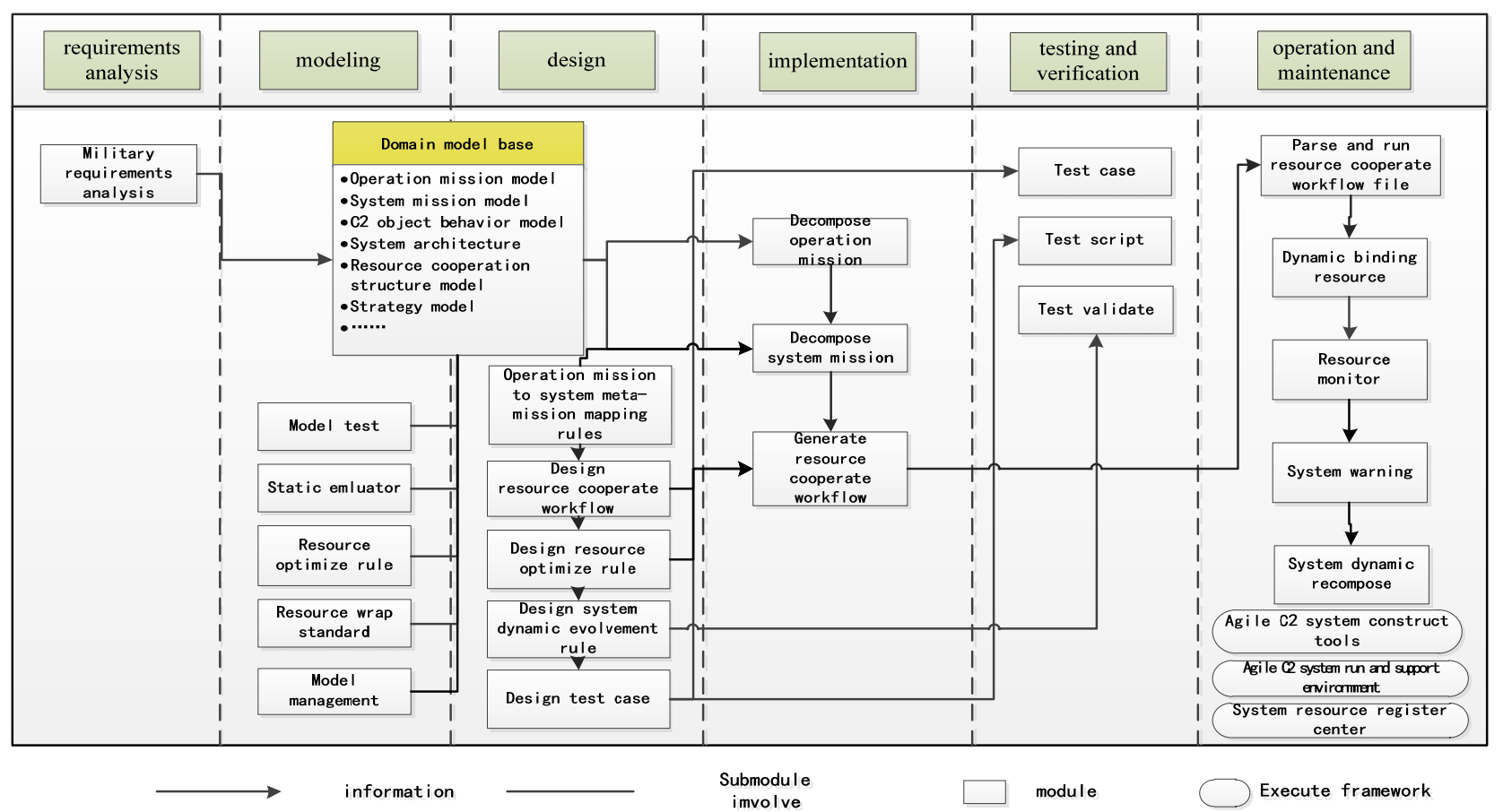

Fig. 2 The overall framework of the model driven agile C2 system 
The mission model, the $\mathrm{C} 2$ object behavior model, and the system resource capability model are studied, and the model's components and the relation types are analyzed, design model's description method and criterion, build dynamic mapping of mission and system resource capability. Based on the model of the object behavior model, the operation process of the accused is abstracted, and the application mode of the system is abstracted, and the dynamic organization of system resources is realized.

Domain model. The main research includes the operations mission model, the $\mathrm{C} 2$ object behavior model, the system resource model, and the relational model, form repository of agility C2 system. First sort out the typical operations missions, set up the classification system of operation mission, extract the operations missions, and then model the operation mission (which means to be unable to carry out the decomposition of the mission), form dictionary of operations mission. At the same time, from the system mission information needs, function description, mission execution constraints and related missions described in the system of the system missions (which can be completed by a single system resource system missions) to model, form a system mission dictionary. And then, from the aspects of object behavior ability, dependence condition, behavior way and behavior state, the object behavior is modeled, and the relationship between the mission model and the system mission model is studied.

Operations missions to system element mission dynamic mapping. Operation mission to system meta-mission dynamic mapping process is shown in Figure 3, Based on the research results of the basic model, the relationship between the mission ability and the behavior ability of the object behavior model is studied; the mission-C2 object relationship model is built. Then, the mapping of attributes such as behavior ability description, depends and mission information needs, function description of system meta-mission model is established, formed C2 object-system meta-mission relationship model, and form mission-system meta-mission relationship model finally.

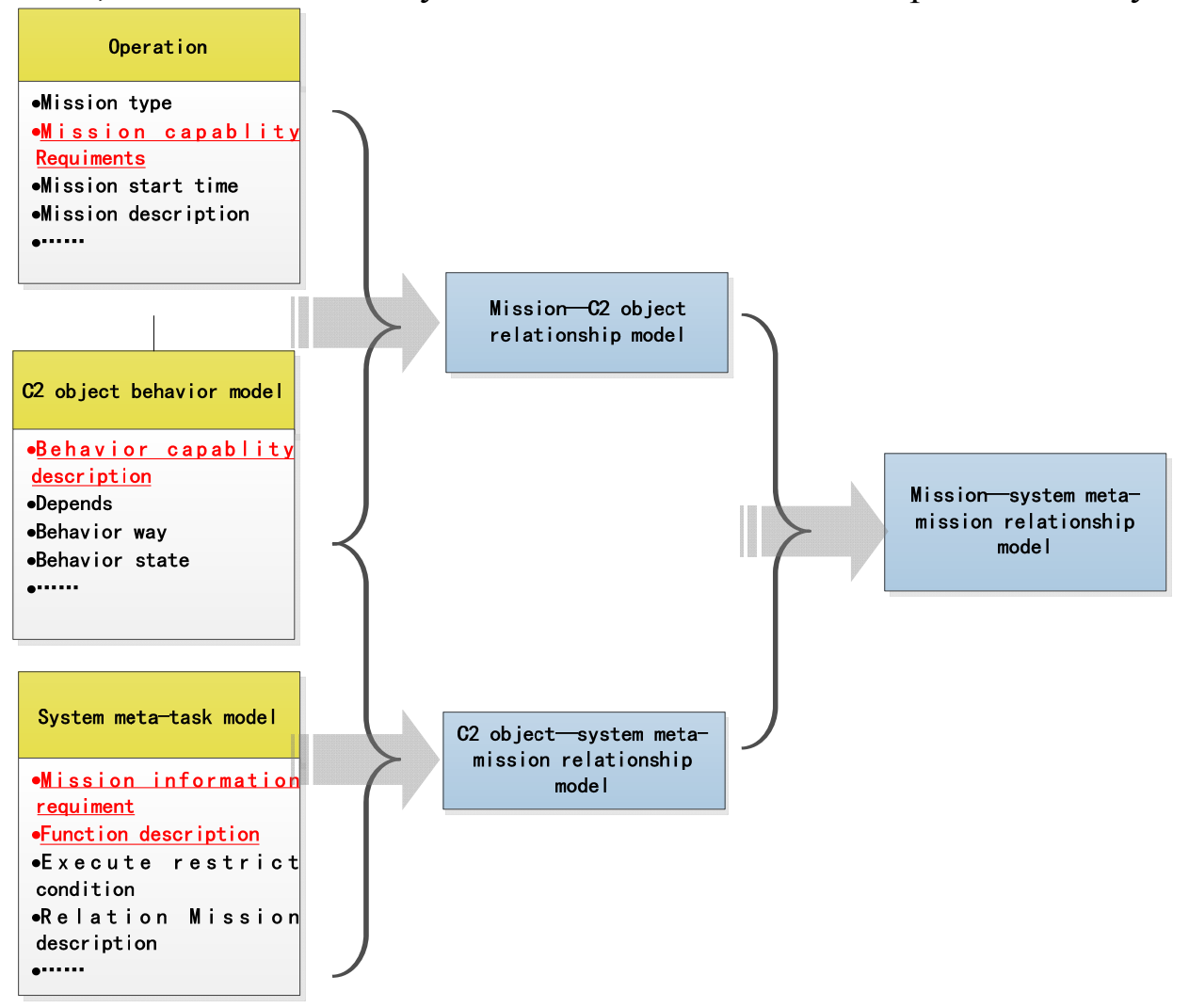

Fig. 3 Operation mission to system meta-mission dynamic mapping

Construct method of Agile C2 system. The principle of agile C2 system is shown in Figure 4, the core of which are operations mission driven and the system resource cooperative structure adjusting, 
which mainly includes the construction framework and repository of agile $\mathrm{C} 2$ system. The framework includes four parts, which includes the operations mission decomposition, the system mission mapping decomposition, the system resource cooperative work flow generation, and the running support environment. The running support environment is mainly to load resources collaborative workflow file and resource monitoring and management. In this paper, we focus on the research on the mechanism and method of the agile charge system, and the research on the operation support environment is not included.

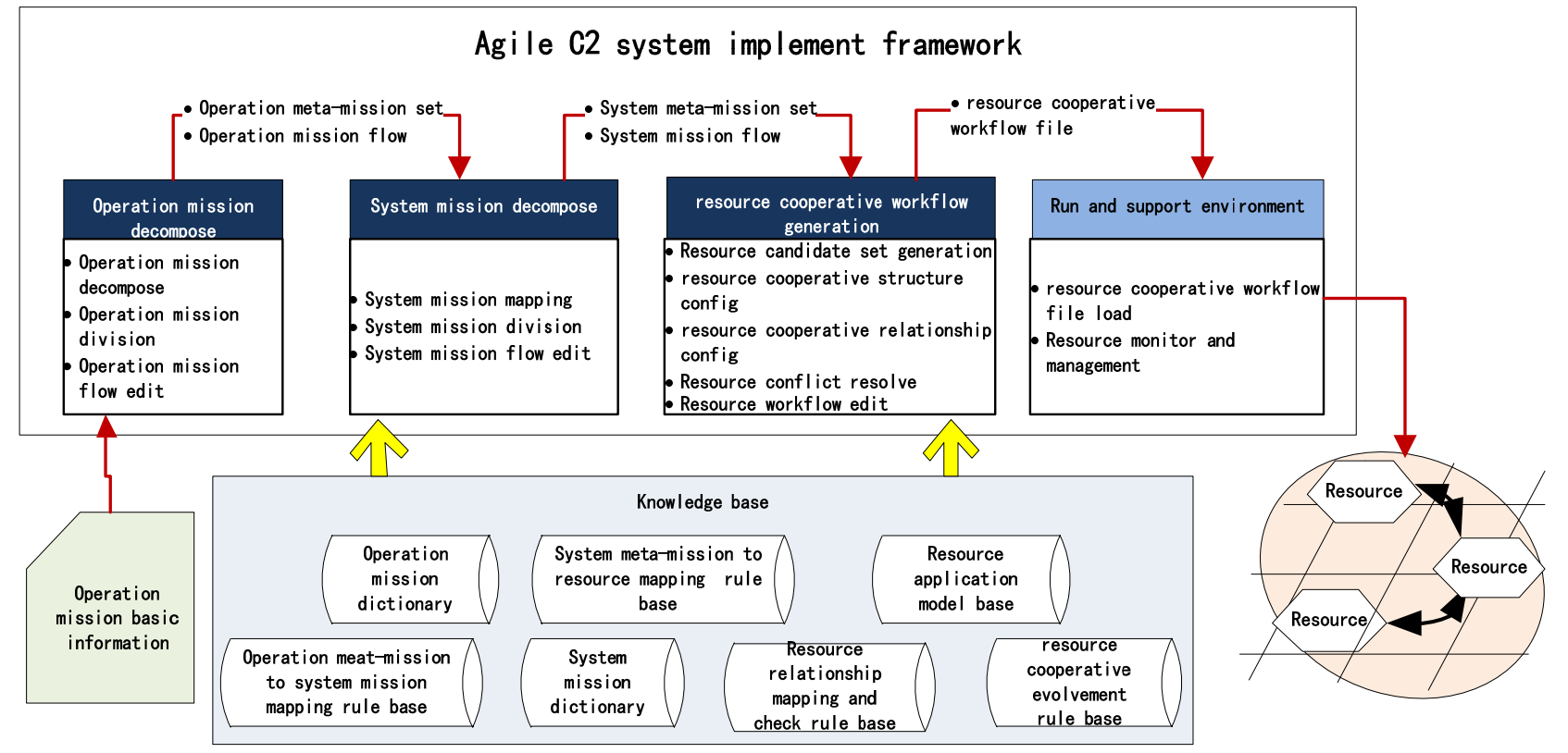

Fig. 4 The principle of agile C2 system

The implementation steps of the agile $\mathrm{C} 2$ system are as follows:

first of all, according to operation mission list and mission model based operation mission dictionary, operation mission are decomposed to operation meta-mission set and operation mission workflow;

then according to the operation meta-mission to system mission mapping rules in repository, mapped the operation meta-missions to the corresponding system missions, according to system mission dictionary and mission model decomposition to system meta-mission, form a system mission flow;

According to the system meta-mission to resource mapping rules in repository, mapped system meta-missions to system resources; configure according to the application mode of organizational resources of resources collaborative structure and relationship.

The resource collaborative workflow conflict resolution check. To edit the corresponding adjustment, to generate resources collaborative workflow file and send to the running support environment;

The runtime support environment load resource collaborative workflow file, generate and run the agile $\mathrm{C} 2$ system.

\section{Summary}

Agile command and control will become the basic force, agility is directly related to the ability of the network centric warfare, and want to be independent of the metric. C2 system as the "nerve center", also need to have agility. In this paper, we propose a new method to construct agile C2 system based on model driven theory, the core of which are operations mission driven and the system resource cooperative structure adjusting. Through the operations mission to the system meta-mission 
dynamic mapping method, the future distribution of system resources in the grid can be coordinated by the workflow to satisfy system capability of operations mission.

\section{References}

[1] D.S. Alberts, Agility AdvantageAdvantage: A Survival Guide for Complex Enterprises and Endeavors, Washington[M], DC: CCRP Publications Series, 2011.

[2] David S. Alberts. Agility Quotient[J]. 19th ICCRTS.2014.

[3] LIU Hai-xiao. Design and Adjust Methodology of Command and Control Organization[D].Chang Sha: 2010. (in Chinese)

[4] YANG Yao, XIU Bao-xin, YANG Ting-ting, ZHANG Wei-ming, BAO Wei-dong. R esearch on R obustness of Agile C2 Organizations[J]. Command Control \& Simulation,2014,4. (in Chinese)

[5] Lan Yushi. Understanding the network-centered command information system[J].Command Information System and Technology, 2010, 1(1):1-4.(in Chinese) 